\title{
Frequency of antiepileptic drugs and response change in pediatric patients receiving 2 or more antiepileptic drugs
}

Abla M. Albsoul-Younes, BSc, PhD, Amira T. Masri, MD, PhD, Lobna F. Gharaibeh, BSc, MSc, PhD, Amer A. Murtaja, BSc, MSc, Abdelkarim A. Al-Qudah, $M D, A B C N$.

\begin{abstract}
الأهداف : دراسة مدى التغييرات التي تطرأ على الأدوية المضادة

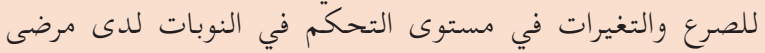

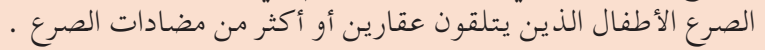

المنهجية : أجريت دراسة متابعة مستقبلية في عيادات طب الأطفال

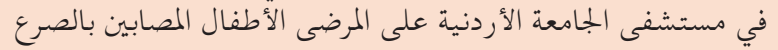

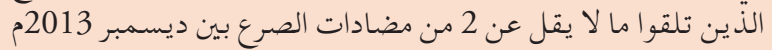

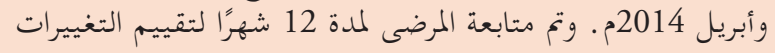

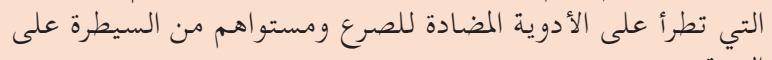
النوبة.

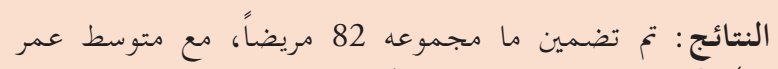

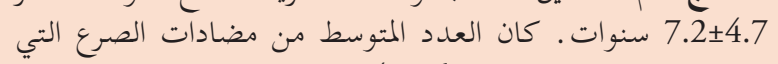

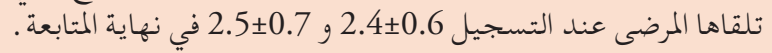

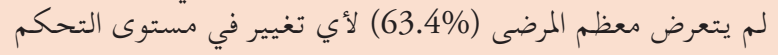

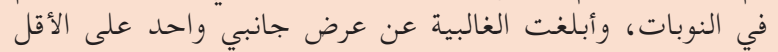

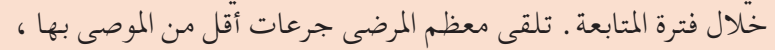

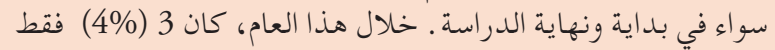

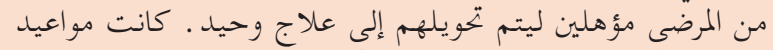

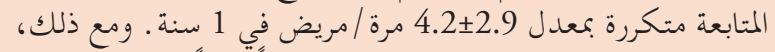

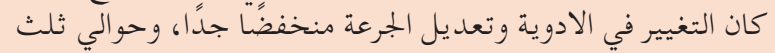

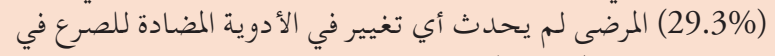

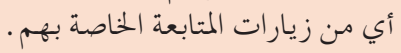

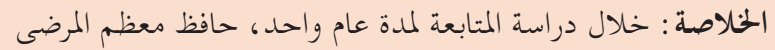

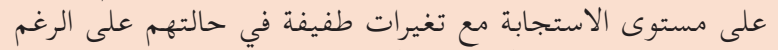

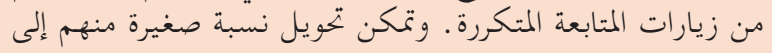

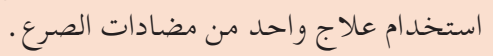

Objectives: To investigate the frequency of changes in antiepileptic drugs (AEDs) use, as well as concomitant changes in the degree of seizure control in pediatric patients, who are receiving 2 or more AEDs.

Methods: A prospective follow-up study at Jordan University Hospital's pediatric neurology clinics was conducted on epileptic pediatric patients receiving at least 2 AEDs between December 2013 and April 2014. Patients were followed for 12 months.

Results: A total of 82 patients were included, with a mean age of $7.2 \pm 4.7$ years. The mean number of AEDs received by patients at enrollment was $2.4 \pm 0.6$, and $2.5 \pm 0.7$ after follow-up. Most patients (63.4\%) experienced no change in seizure control, and the majority reported at least one adverse drug reaction. Most patients received lower doses than recommended, both at the beginning and end of the study. During the year, only 3 patients (4\%) were eligible for dose tapering, which would then be converted to monotherapy. Follow-up appointments average was $4.2 \pm 2.9$ visits/patients in one year. The frequency of medication changes and dose adjustment was very low, about one-third (29.3\%) of patients requiring no change in $\mathrm{AEDs}$ during any follow-up visits.

Conclusion: During the one year follow-up study, most patients on polytherapy maintained their level of response to the AEDs, with minimal changes in their regimen despite frequent follow-up visits. Only a small percent could be converted to AEDs monotherapy.

\section{Neurosciences 2020; Vol. 25 (4): 269-275 doi: 10.17712/nsj.2020.4.20190113}

From the Department of Biopharmaceutics and Clinical Pharmacy (Albsoul-Younes, Gharaibeh, Murtaja), School of Pharmacy, Department of Pediatrics (Masri, Al-Qudah), School of Medicine, University of Jordan, Amman, Jordan

Received 13th February 2020. Accepted 16th May 2020.

Address correspondence and reprint request to: Dr. Abla AlbsoulYounes, Department of Biopharmaceutics and Clinical Pharmacy, School of Pharmacy, The University of Jordan, Amman, Jordan. E-mail:ablabsoul@yahoo.com

ORCID ID: https://orcid.org/0000-0002-4749-4066 
$\mathrm{E}_{\mathrm{in}}^{\mathrm{p}}$ pilepsy is the most common neurological disorder in children. ${ }^{1}$ Despite the increase in the number of antiepileptic drugs (AEDs), about one third of patients with childhood epilepsy continue to have seizures. ${ }^{2}$ Successful management of epilepsy depends on many factors, including the cause of epilepsy, type of epilepsy, and AED-related issues, such as: dosing, compliance, side effects, monitoring, and pharmacokinetics. ${ }^{3,4}$ Most AEDs have a suboptimal patient tolerability profile (with a wide range of side effects); the second-generation AEDs may be better tolerated than the traditional AEDs, but are not actually more effective. ${ }^{5}$

Due to the complexity of classifying seizures, as well as the difficulty in choosing the most appropriate AED, many patients must deal with drug therapy changes over the course of their treatment/management. ${ }^{5-7}$ It has been estimated that approximately $50 \%$ of patients who are newly diagnosed with epilepsy become seizure-free for at least 12 months on their first AED. ${ }^{7}$ However, about one-third receive 2 or more AEDs, while continuing to experience seizures. ${ }^{5}$ In general, patients requiring multiple AEDs to manage their epilepsy represent the more resistant group of patients. This group must be closely followed to optimize their AED regimen; ${ }^{8}$ as the change in drug therapy might be more likely, but improvement is expected to be limited. Cited reasons for lack of improvement include incorrect diagnosis, inappropriate choice of AED and/or dose, in addition to lack of adherence. Data regarding follow-up studies of pediatric patients on AED polytherapy is scarce. ${ }^{9,10}$ The aim of the current study is to conduct a short-term (12 months) follow-up study of the change in AEDs, as well as the response in a subgroup of pediatric epileptic patients receiving AED polytherapy.

Methods. Study design. This is part 2 of the Jordanian pediatric epilepsy project, while part 1 is a cross-sectional study of patterns of AED use, and types of epilepsy. ${ }^{11,12}$ This part is a prospective, observational, single-center short-term (12 months) follow-up study of pediatric epileptic patients who are maintained on polytherapy (2 or more AEDs).

Setting. This study was conducted at Jordan University Hospital (JUH), which is the tertiary care

Disclosure. This study was funded and supported from the Scientific Research Support Funds, Ministry of Higher Education and Scientific Research, Amman, Jordan. hospital of The University of Jordan. JUH is one of 2 university hospitals in Jordan, and is situated in Amman, the capital and central city of Jordan: it is considered to be a referral hospital that mainly serves the central and southern parts of Jordan.

Inclusion and exclusion criteria. All patients from 6 months to 18 years, with a final diagnosis of epilepsy according to the 2010 proposed report of The International League Against Epilepsy (ILAE) Commission, received the "Classification and Terminology of Epileptic Seizures." 13 Diagnosis was carried out by 2 pediatric neurologists at JUH, who are coauthors of this work. For this study, intractability was defined as having no 2-month remission identified during the one-year period, despite adequate medical treatment. ${ }^{14}$ All patients who were febrile, acutely symptomatic, with isolated neonatal seizures were excluded. ${ }^{15}$

Ethical approval. This study was conducted according to principles of Helsinki Declaration. The study was initiated after ethical approval was obtained from the Institutional Review Board Committee at JUH. Eligible patient guardians were asked for written consent for the enrollment of their children. With consent, we collected pertinent information using a case report form. Clinical records were used to fill in all relevant patient medical history. The enrollment period lasted for 5 months, December 2013 until April 2014, with follow-up lasting until April 2015. During this period, each patient was seen at his/her regular follow-up visit with pediatric neurologists, as scheduled by the treating physicians.

Classification of AEDs. When classifying AEDs, the following criteria are used: those that were in clinical use before 1993 are considered to be older generation antiepileptic drugs, which include carbamazepine, phenytoin phenobarbital, ethosuximide, primidone,

Table 1 - General characteristics of the patients $(\mathrm{N}=82)$.

\begin{tabular}{lc}
\hline Characteristics & n (\%) \\
\hline Gender & \\
Male & $50(61)$ \\
Female & $32(39)$ \\
Types of seizures & \\
Generalized & $21(25.6)$ \\
Focal seizures & $33(40.2)$ \\
Spasms & $12(14.6)$ \\
Mixed seizures & $16(19.5)$ \\
Intractability & \\
Yes & $45(54.9)$ \\
No & $37(45.1)$ \\
\hline
\end{tabular}


Table 2 - Number of antiepileptic drugs (AEDs) prescribed per patient, number of newer generation AEDs used*, and level of seizure control achieved in patients at enrollment and at the end of one-year follow-up $(\mathrm{N}=82)$.

\begin{tabular}{|c|c|c|}
\hline Variables & $\begin{array}{l}\text { At the start of follow-up, } \\
\mathrm{n}(\%)\end{array}$ & $\begin{array}{l}\text { At the end of follow-up, } \\
\text { n (\%) }\end{array}$ \\
\hline \multicolumn{3}{|c|}{ Number of AEDs prescribed to control seizures } \\
\hline One AED & $0(0)$ & $3(3.7)$ \\
\hline 2 AEDs & $54(65.9)$ & $44(53.7)$ \\
\hline $3 \mathrm{AEDs}$ & $23(28.0)$ & $26(31.7)$ \\
\hline 4 AEDS & $5(6.1)$ & $9(11)$ \\
\hline \multicolumn{3}{|c|}{ Number of newer generation AEDs prescribed per patient } \\
\hline None & $28(34.1)$ & $28(34.1)$ \\
\hline One AED & $40(48.8)$ & $34(41.5)$ \\
\hline 2 AEDs & $13(15.9)$ & $20(24.4)$ \\
\hline 3 AEDs & $1(1.2)$ & $0(0)$ \\
\hline \multicolumn{3}{|c|}{ Level of seizure control (\%) } \\
\hline$<25 \%$ & $13(15.9)$ & $12(14.6)$ \\
\hline $25<50 \%$ & $4(4.9)$ & $6(7.3)$ \\
\hline $50<75 \%$ & $11(13.4)$ & $15(18.3)$ \\
\hline $75<100$ & $21(25.6)$ & $20(24.4)$ \\
\hline $100 \%$ & $33(40.2)$ & $29(35.4)$ \\
\hline
\end{tabular}

*When classifying AEDs, the following criteria were used: AEDs that were in clinical use before 1993 were considered to be older generation antiepileptic drugs, which include carbamazepine, phenytoin phenobarbital, ethosuximide, primidone, clonazepam, and valproic acid. While, AEDs approved for clinical use after 1993 were considered newer-generation drugs, including tiagabine, felbamate, gabapentin, oxcarbazepine, zonisamide, lamotrigine, topiramate, and levetiracetam ${ }^{16}$

clonazepam, and valproic acid. AEDs approved for clinical use after 1993 are considered newer-generation drugs, including tiagabine, felbamate, gabapentin, oxcarbazepine, zonisamide, lamotrigine, topiramate, and levetiracetam. ${ }^{16}$

AEDs dose calculation. Patients' AED doses were assessed at enrollment, and at the end of the follow-up period. For each drug dose, we made a comparison with the recommended dose: this was dependent on age, weight, and type of epilepsy, as per the pediatric patient's condition. ${ }^{17}$ Level of seizure control with AED therapy was assessed as such: with $100 \%$ control, we defined it as the absence of seizures in the last 3 months. Lower responses were judged on the percent of reduction in number of seizures, as per home caregiver assessment, which was divided into 5 categories, from no control to complete control: no control $(<25 \%)$, poor control: ( $25 \%$ to $<50 \%)$, intermediate control $(50 \%$ to $<75 \%)$, good control $(75 \%$ to $<100)$, and complete control (100\%).

Statistics. Descriptive statistics were used to present patient characteristics. Frequency and percentage were implemented to summarize categorical and quantitative
Table 3 - Frequency of adverse drug reactions reported by patients (82) and/or patient' caregivers during follow-up visits.

\begin{tabular}{lc}
\hline Type of adverse drug reaction & $\mathbf{n}(\%)^{*}$ \\
\hline Nervousness and irritability & $43(52.4)$ \\
Reduced appetite & $19(23.1)$ \\
Sedation & $16(19.5)$ \\
Weight gain & $16(19.5)$ \\
Hyperactivity & $15(18.3)$ \\
Anxiety & $6(7.3)$ \\
Behavioral change & $6(7.3)$ \\
Hyperthermia & $6(7.3)$ \\
Somnolence & $6(7.3)$ \\
Gait problems & $2(2.4)$ \\
Insomnia & $2(2.4)$ \\
Memory loss & $2(2.4)$ \\
Rash & $2(2.4)$ \\
Weight loss & $2(2.4)$ \\
\hline *The following adverse drug reactions were reported by only one patient: \\
$\quad$ cognitive impairment, constipation, drooling, increased appetite, \\
increased blood pressure, increased heart rate, psychomotor slowing, \\
shortness of breath, tremor, and urinary incontinence. Some patients \\
$\quad$ reported more than one adverse drug reaction at a time \\
\hline
\end{tabular}

data, using means and standard deviations. The paired t-test assessed statistical differences in continuous data among paired groups, while the independent t-test assessed statistical differences in continuous data among unpaired groups. For all statistical tests, $p$-values less than 0.05 were considered statistically significant. All statistical analyses used $\mathrm{IBM}^{\odot}$ SPSS ${ }^{\oplus}$ Statistics, v. 20 (Armonk, NY, USA).

Results. Three hundred eleven patients with a diagnosis of epilepsy, who used AEDs, were screened during the study period. Of these, 63\% used monotherapy at the time of their recruitment and were not included in the study. Only 103 fulfilled inclusion criteria and were initially invited to be in the study; however, only 82 patients consented and finished the 12-month follow up. The mean age of patients at enrollment was $7.2 \pm 4.7$ years, with the mean onset age of epilepsy as $2.82 \pm 3.30$ years. Most patients were males $(61 \%)$. More than half of the enrolled patients had intractable seizures at enrollment and throughout the study. More than half $(54.9 \%)$ had parents who were related (first- through third-degree relatives). About $40 \%$ of patients had focal seizure disorder, and a quarter had generalized seizure disorder.

Most patients were on dual AED therapy (65.9\%); however, as follow-up progressed, more patients received triple and quadruple therapy. The mean number of medications at enrollment was $2.39 \pm 0.62$, 
Table 4 - Average doses (mg) of antiepileptic drugs used in pediatric patients (82) at the beginning and end of the one year follow-up.

\begin{tabular}{|c|c|c|c|c|c|c|}
\hline \multirow{2}{*}{ Drug Name } & \multicolumn{2}{|c|}{ Frequency (n) } & \multicolumn{2}{|c|}{ Average Doses $($ Mean $\pm S D)$} & \multicolumn{2}{|c|}{ Min-Maximum Dose } \\
\hline & Start & End & Start & End & Start & End \\
\hline Valproic Acid & 57 & 54 & $567.5 \pm 342.7$ & $626.4 \pm 420.9$ & $200-2000$ & $100-2000$ \\
\hline Levetiracetam & 34 & 30 & $795.6 \pm 479.8$ & $893.3 \pm 548.2$ & $150-2000$ & $150-2000$ \\
\hline Carbamazepine & 30 & 30 & $330.7 \pm 160.1$ & $364.7 \pm 197.1$ & $120-700$ & $40-800$ \\
\hline Clonazepam & 24 & 2 & $1.1 \pm 1.2$ & $1.7 \pm 3.6$ & $0.2-6$ & $0.1-20$ \\
\hline Topiramate & 23 & 23 & $86.4 \pm 54.2$ & $71.7 \pm 52.3$ & $25-200$ & $12.5-200$ \\
\hline Phenobarbital & 16 & 10 & $46.0 \pm 27.1$ & $46.5 \pm 18.0$ & $15-90$ & $15-75$ \\
\hline Lamotrigine & 8 & 10 & $128.1 \pm 71.3$ & $113.8 \pm 95.5$ & $25-225$ & $25-300$ \\
\hline Vigabatrin & 7 & 8 & $517.9 \pm 292.5$ & $703.1 \pm 312.9$ & $125-1000$ & $125-1000$ \\
\hline Oxcarbazepine & 1 & 1 & $720 \pm 0$ & $720 \pm 0$ & $720-720$ & $720-720$ \\
\hline
\end{tabular}

but by the end of follow-up, it was $2.50 \pm 0.74(p=0.14)$. General patient characteristics at enrollment are shown in Table 1.

At enrollment, over half the study participants demonstrated a $75 \%$ level of seizure control or more. Approximately $63 \%(n=52)$ of the study group showed no change in level of seizure control in this period, with $21(25.6 \%)$ patients maintaining 100\% seizure control. A change in level of control was observed in 30 patients, out of which 15 (18.3\%) experienced a deterioration in seizure control, and $15(18.3 \%)$ experienced an improvement.

Twenty-one patients, maintaining 100\% control through the study period, were further investigated; we noted that 3 underwent dose tapering and converted to monotherapy by the end of the study (Table 2). Comparing the average number of AEDs in patients who demonstrated $100 \%$ control vs. the other groups, there was no significant difference $(p=0.521)$ between the 2 groups. As expected, the average number of medications at the end of the study was significantly reduced in the $100 \%$ responders to 2.19 , while they increased in the other groups to $2.61 \quad(p=0.026)$. The same applied to the number of follow-up visits, including follow-up visits with medication changes. The responders had significantly lower rates of changes and number of visits, with the average number in the 12 -month period at 3.14 in $100 \%$ responders, compared to 4.57 in the other groups $(p=0.018)$; the average number of follow-up visits that showed a change was 0.86 in $100 \%$ responders vs. 1.1 in the other patient groups $(p=0.01)$. Fifteen patients who experienced a decrease in response were investigated for the possibility of decreased drug levels, but test results suggested that AED serum levels were within an acceptable range. From a total of 8 patients on valproic acid, 6 experienced a decrease in response; however, when therapeutic drug levels were tested, all serum levels were within range. Ten patients were receiving carbamazepine, out of which 7 experienced a decrease in the level of control. The serum level of carbamazepine was found to be within a therapeutic range for 6 of the patients. Furthermore, 2 of the 3 phenobarbitone users experienced a decrease in seizure control, although serum levels were maintained within range.

Adverse drug reactions were assessed at every visit. Fifty-nine patients $(72.0 \%)$ reported at least one adverse drug reaction (ADR) in follow-up visits. Various ADRs were reported, with some patients suffering from more than one reaction at the same time (Table 3). Reported ADRs were collected from 128 follow-up visits, given a total of 345 follow-up visits (37.1\%). Steps were taken to manage the adverse drug reactions in only $19(14.8 \%)$ of all reactions, which consisted of either discontinuation or a decrease in the AED dose.

Although the total number of visits during the 12-month follow-up was 347, with an average of $4.23 \pm 2.90$, almost one-third of patients had no change in medication during any follow-up visits $(n=24,[29.3 \%])$. The rate of AED alteration and dose adjustment was noted to be slow. In the group of patients who experienced at least one change during any follow-up visit, only 139 (40.1\%) of the follow-up visits involved a change in medication. The modifications in follow-up visits involved a change in one AED or more, with the total number of changes in AEDs being 176 (more than one change could occur per visit). Changes involved an increase in medication dose $(n=78,[44.3 \%])$, a decrease in dose $(n=39,[22.2 \%])$, the addition of a new medication $(n=32,[18.2 \%])$, or discontinuation of a medication $(n=27,[15.3 \%])$. Patients with intractable seizures had slightly more frequent follow-up visits, as well as more medication changes than other patients. Yet, there was no significant increase in the number of follow-up visits for intractable seizures, vs. those with non-intractable 
seizures $(4.53 \pm 3.24$ vs. $3.81 \pm 2.49, p=0.269)$, and no significant increase in the average number of follow-up visits requiring a change $(1.91 \pm 1.77)$, compared to those with non-intractable seizures $(1.43 \pm 1.66, p=0.2)$.

Most pediatrics patients were using combination of older generation drugs (Table 1 and Table 4). Valproate followed by carbamazepine and levetiracetam were the drug most used in (Table 4). In general, patient doses were less recommended, with average medication doses illustrated in Table 4. Eighty-two patients enrolled in this study used a total of 197 drug doses at the beginning of the study, and 196 doses by the end of the follow-up. There were 131 incidents in which the AED doses at enrollment were less than the recommended doses $(67 \%)$, compared to $123(63 \%)$ at the end. Only $51(26 \%)$ doses at enrollment, compared to $65(33 \%)$ at the end, were within the recommended range. The number of higher doses vs. recommended doses were more frequent at the start than at the end of follow-up: $15(7 \%)$, vs. $8(4 \%)$.

In the follow-up period, $24(29.3 \%)$ patients experienced at least one hospitalization, with a total of 41 admissions; these were mainly due to uncontrolled seizures $(n=30[73.2 \%])$, followed by other hospital admissions to perform EEG or receive steroid or adrenocorticotrophic hormone $(\mathrm{ACTH})$ therapy $(\mathrm{n}=4$ [9.8\%]), or admission for further investigation $(n=3$ $[7.3 \%])$.

Discussion. Epilepsy is a chronic disease; and its treatment is usually long-term. Patients receiving more than one AED are usually the more refractory group. Consistent with the previously reported data on pediatric epileptic patients receiving polytherapy more than half of pediatric patients included in this study had intractable seizures. Focal seizure diagnosis was the most reported seizure type. Most patients were males (61\%) and were diagnosed with epilepsy a few years earlier. ${ }^{8-10}$ In agreement with previous reports older generation AEDs were more frequently used than newer generation drugs. ${ }^{11}$ This study looked at a one-year interval of the pediatric epileptic patient's journey in terms of managing the disease. At the end of 12-months follow-up, there was no significant change in response for most patients. Tapering of AED is recommended in an epileptic patient who is seizure-free for some time, usually 2 to 5 years. However, this action can be complicated by the recurrence of seizures after AED withdrawal. ${ }^{18}$ We found that 21 of the enrolled patients at the end of the study maintained 100\% response, but none had AEDs withdrawn completely, and only 3 were converted to monotherapy after the follow-up period. The average number of medications in this patient group was significantly reduced, while maintaining a within range response.

With each decision for AED change, the physician must balance response and tolerability (i.e., the patient's quality of life), and those with intolerable side effects to AEDs are at higher risk for drug therapy changes. ${ }^{6,7,10}$ In this study, the majority of patients reported AED-related side effects. The most frequently reported were irritability and nervousness. Most patients used less than the average recommended dose, which may be due to the physician's attempt to minimize side effects. Responding to patient complaints about adverse drug reactions is important, in that a parent of a child with an adverse drug reaction may decide to stop treatment, or decrease the dose, without proper action by the physician. It has been suggested that patient compliance to prescribed drug dosing regimens could decline substantially between clinic visits and drug level tests. Some patients decide to decrease or stop medication between visits, but may still register within therapeutic range after drug level testing. ${ }^{19}$ This can partly explain discrepancies in most drug levels remaining in the therapeutic range, although there was a deterioration in the level of seizure control in a subgroup of patients.

Patients with intractable seizures are at higher risk for drug therapy changes. ${ }^{6,7,10}$ More than half the patients in this study had intractable seizures, but continued to have them without any association between intractability and change in response.

Slow dose titration in pediatric patients was advocated to decrease side effects and to optimize treatment. ${ }^{20}$ Consistent with previous work, a high follow-up rate was reported in this study - but the frequency of medication change was low, and average AEDs doses were lower than the recommended doses. In addition, this study reported a large percentage of patients on less than the recommended doses, which could explain most patients not having 100\% response, along with a lack of significant difference between response at the start and end of follow-up.

The older generation AEDs are the most frequently used drugs, even for patients on polytherapy. In line with other studies, valproic acid was the most commonly used older-generation AED, followed by the new-generation AED levetiracetam. ${ }^{21}$

Conclusions and recommendations. Most patients on 2 or more medications maintained their level of response to the AEDs after one year of follow-up. Minimal changes were made in their AEDs regimens despite frequent follow-up visits. Only a small percent could be converted to AEDs monotherapy. However, 
this study found that a group of patients had a decrease in response, despite AEDs at the recommended therapeutic level. Most patients received less than the recommended dose of AEDs: this is acceptable when they achieve $100 \%$ response, but not those with suboptimal control. The effectiveness of therapeutic drug monitoring in treatment decisions should be explored, as well as the importance of dose titration to attain the desired response. It also suggests that individualizing treatment decisions is essential, e.g., when to adjust the dose, switch AEDs, or add a new medication. Moreover, emphasis must focus on making this decision in a timely manner, to reflect patients' improvement and/ or improved tolerability to treatment. Despite being a relatively short period of follow-up. This study has the strength of being a prospective study documenting both AEDs dosing regimen and response changes.

Limitations. This was an observational study, and drug level testing was available for only a limited number of medications. Conclusions regarding therapeutic range and response could not be conclusive, as a result. The low sample size and short period of follow-up, especially for epilepsy, in which change is very slow, did not facilitate conclusions about drug tapering or titration in this group.

Although slow dose titration is desirable, physicians must balance this with some rapid decisions to respond to improvement on the patient's side. ${ }^{19,20}$ Despite that side effects were assessed at each visit, adherence was not checked. Therefore, it was not possible to conclude that the presence of an adverse effect affected drug compliance or subsequent response. Patients should be counseled about expectations and the acceptance of a certain number of side effects, in the hope of achieved improvement during treatment. Future studies to assess patient compliance at each visit, with a longer follow-up period and larger sample, is recommended.

After a trial of 2 drugs, the possibility of other drugs achieving seizure control is low. In those with intractable epilepsy, the goal of treatment would not be zero seizures, but would aim for the best quality of life, or the least number of seizures with the fewest side effects; patients must accept some seizures, as 30\% will be resistant to whatever drugs are tried. ${ }^{8-10}$ Since this study assessed only one year, other drugs and dose trials could have been attempted in advance; many patients were eligible for surgery, vagal nerve stimulation, or a ketogenic diet, but they would likely not have been able to afford these options.

Implications of findings for future research. Future studies to assess this group of patients should include larger sample size and longer period of time. Furthermore, assessment of pediatrics patient's compliance as well as acceptability of the adverse effects the drug is causing at each visit would allow making a more informed decision regarding AED choices by the physician.

Acknowledgment. We would like to thank American Manuscript Editors (www.americanmanuscripteditors.com) for English language editing.

\section{References}

1. Bashiri FA. Childhood epilepsies: What should a pediatrician know? Neurosciences (Riyadh) 2017; 22: 14-19.

2. Naimo GD, Guarnaccia M, Sprovieri T, Ungaro C, Conforti FL, Andò S, et al. A Systems Biology Approach for Personalized Medicine in Refractory Epilepsy. Int J Mol Sci 2019; 20: E3717.

3. Russo A, Posar A, Conti S, Parmeggiani A. Prognostic factors of drug-resistant epilepsy in childhood: An Italian study. Pediatr Int 2015; 57: 1143-1148.

4. Verrotti A, Tambucci R, Di Francesco L, Pavone P, Iapadre G, Altobelli E, et al. The role of polytherapy in the management of epilepsy: suggestions for rational antiepileptic drug selection. Expert Rev Neurother 2020; 20: 167-173.

5. Park KM, Kim SE, Lee BI. Antiepileptic Drug Therapy in Patients with Drug-Resistant Epilepsy. J Epilepsy Res 2019; 9: 14-26.

6. Joshi R, Tripathi M, Gupta P, Gulati S, Gupta YK. Adverse effects \& drug load of antiepileptic drugs in patients with epilepsy: Monotherapy versus polytherapy. Indian J Med Res 2017; 145: 317-326.

7. Martínez-Lizana E, Gil-Lopez F, Donaire A, Aparicio J, Brandt A, Carreño M. Outcome of treatment changes in patients with drug-resistant chronic epilepsy: A tertiary center experience. Epilepsy Res 2017; 136: 97-102.

8. Nair DR. Management of Drug-Resistant Epilepsy. Continuum (Minneapolis, MN, USA) 2016; 22: 157-172.

9. Plevin D, Jureidini J, Howell S, Smith N. Paediatric antiepileptic polytherapy: systematicreview of efficacy and neurobehavioural effects and a tertiary centre experience. Acta Paediatr 2018.

10. Egunsola O, Choonara I, Sammons HM, Whitehouse WP. Safety of antiepileptic drugs in children and young people: A prospective cohort study. Seizure 2018; 56: 20-25.

11. Albsoul-Younes A, Gharaibeh L, Murtaja AA, Masri A, Alabbadi I, Al-Qudah AA. Patterns of antiepileptic drugs used in epileptic pediatric patients in Jordan. Neurosciences (Riyadh) 2016 ; 21: 264-267.

12. Al-Qudah AA, Albsoul-Younes A, Masri AT, AbuRahmah SK, Alabadi IA, Nafi OA, et al. Type and etiology of pediatric epilepsy in Jordan. A multicenter study. Neurosciences (Riyadh) 2017; 22: 267-273.

13. Fisher RS, Cross JH, French JA, Higurashi N, Hirsch E, Jansen FE, et al. Operational classification of seizure types by the International League Against Epilepsy: Position Paper of the ILAE Commission for Classification and Terminology. Epilepsia 2017; 58: 522-530. 
14. Camfield PR, Camfield CS. Intractable seizures after a lengthy remission in childhood-onset epilepsy. Epilepsia 2017; 58: 2048-2052.

15. Syvertsen M, Nakken KO, Edland A, Hansen G, Hellum MK, Koht J. Prevalence and etiology of epilepsy in a Norwegian county: A population based study. Epilepsia 2015; 56: 699-706.

16. Sirven JI, Noe K, Hoerth M, Drazkowski J. Antiepileptic drugs 2012: Recent advances and trends. Mayo Clin Proc 2012; 87: 879-889.

17. Lacy CF, Armstrong LL, Goldman MP, Lance LL, editors. Drug Information Handbook: A Comprehensive Resource for All Clinicians and Healthcare Professionals (Lexi Comp's Drug Information Handbooks). 15th Edition. Hudson (Ohio): Lexi-Comp Inc; 2013.
18. Moosa ANV. Antiepileptic Drug Treatment of Epilepsy in Children. Continuum (Minneap Minn) 2019; 25: 381-407.

19. O’ Rourke G, O’ Brien JJ. Identifying the barriers to antiepileptic drug adherence among adults with epilepsy. Seizure. 2017; 45: 160-168.

20. Fishman J, Kalilani L, Song Y, Swallow E, Wild I. Antiepileptic Drug Titration and Related Health Care Resource Use and Costs. J Manag Care Spec Pharm 2018; 24: 929-938.

21. Gupte-Singh K, Wilson JP, Barner JC, Richards KM, Rascati KL, Hovinga C. Patterns of antiepileptic drug use in patients with potential refractory epilepsy in Texas Medicaid. Epilepsy Behav 2018; 87: 108-116.

\section{Ethical Consent}

All manuscripts reporting the results of experimental investigations involving human subjects should include a statement confirming that informed consent was obtained from each subject or subject's guardian, after receiving approval of the experimental protocol by a local human ethics committee, or institutional review board. When reporting experiments on animals, authors should indicate whether the institutional and national guide for the care and use of laboratory animals was followed. 